Preprint typeset in JHEP style - PAPER VERSION

\title{
Instantons, Fermions and Chern-Simons Terms
}

\author{
Benjamin Collie and David Tong \\ Department of Applied Mathematics and Theoretical Physics, \\ University of Cambridge, UK \\ b.p.collie, d.tong@damtp.cam.ac.uk
}

\begin{abstract}
In five spacetime dimensions, instantons are finite energy, solitonic particles. We describe the dynamics of these objects in the presence of a Chern-Simons interaction. For $U(N)$ instantons, we show that the $5 \mathrm{~d}$ Chern-Simons term induces a corresponding Chern-Simons term in the ADHM quantum mechanics. For $S U(N)$ instantons, we provide a description in terms of geodesic motion on the instanton moduli space, modified by the presence of a magnetic field. We show that this magnetic field is equal to the first Chern character of an index bundle. All of these results are derived by a simple method which follows the fate of zero modes as fermions are introduced, made heavy, and subsequently integrated out.
\end{abstract}




\section{Introduction}

In five spacetime dimensions, the self-dual instanton solutions of Yang-Mills theory are finite energy, solitonic particles. Their dynamics is described by quantum mechanics on the instanton moduli space $\mathcal{M}$. The purpose of this paper is to explain how this dynamics is altered by the presence of a $5 \mathrm{~d}$ Chern-Simons (CS) term.

This topic has received some interest of late, motivated in part by an old observation of Atiyah and Manton which relates instantons to Skyrmions [1]. This idea has recently found a dynamical realization within the context of holographic QCD $[2,3]^{1}$. In this framework, a $U\left(N_{f}\right)$ Yang-Mills theory in a five-dimensional warped background reduces at low-energies to the Skyrme model with $N_{f}$ flavours. The 5 d theory includes CS interactions which play a key role in the story and, for $N_{f} \geq 3$, give rise to the WZW term of the Skyrme model. Baryons in this model arise from instantons in 5d and have been studied in a number of papers [7-11].

Here we study the dynamics of instantons in flat five-dimensional Minkowski space. To derive the effects of the CS interactions on the instanton dynamics, we use a simple method which has its roots in the earlier, classic work of $[12,13]$. We examine the effects of massive fermions which, once integrated out, generate the CS terms of interest. By following the fate of the fermi zero modes, one arrives at a simple description of the instanton dynamics in terms of geodesic motion, modified by the presence of a background magnetic field on $\mathcal{M}$. In a companion paper [14], we will use this technique to describe the dynamics of vortices in three-dimensional CS theories.

We primarily focus on the dynamics of BPS instantons in $d=4+1 U(N)$ theories with $\mathcal{N}=1$ supersymmetry. In the absence of CS terms, a simple and convenient description of the instanton dynamics is provided by the ADHM quantum mechanics $[15,16]$. Our main result is that the $5 \mathrm{~d}$ CS term has a very simple effect in the ADHM language: it induces a corresponding CS term in the quantum mechanics of the type discussed previously in [17].

We start in Section 2 by reviewing some aspects of the 5 d theory, including the instanton solutions and manner in which fermions induce CS interactions. In Section 3 , we turn to the effective theory of the instanton dynamics, working firstly in the ADHM formalism. We show how fermions induce CS terms in the quantum mechanics. We end with a discussion of $S U(N)$ instantons (as opposed to $U(N)$ instantons), where

\footnotetext{
${ }^{1}$ There are also a number of further proposals which provide different dynamical realizations of the relationship between $5 \mathrm{~d}$ instantons and Skyrmions $[4,5,6]$.
} 
we provide a more geometric description of the instanton dynamics. We show that the magnetic field over $\mathcal{M}$ is given by the first Chern character of the bundle of fermi zero modes.

Note added: In the final stages of preparation of this paper, a preprint appeared on the arXiv which contains substantial overlap with our work [18].

\section{Instantons in 5d Chern-Simons Theories}

In this section we consider the five-dimensional theory with $\mathcal{N}=1$ supersymmetry (i.e. 8 supercharges) and gauge group $G=U(N)$. The vector multiplet contains a gauge field $A_{\mu}$, a real adjoint scalar $\phi$ and an adjoint Dirac fermion $\lambda$. In the absence of a Chern-Simons interaction, the bosonic part of the Lagrangian is given by the familiar expression $^{2}$

$$
\mathcal{L}_{Y M}=\frac{1}{e^{2}} \delta_{a b}\left(-\frac{1}{4} F_{\mu \nu}^{a} F^{b \mu \nu}-\frac{1}{2} \mathcal{D}_{\mu} \phi^{a} \mathcal{D}^{\mu} \phi^{b}\right)
$$

When $\langle\phi\rangle=0$, the gauge symmetry is unbroken and this theory admits the familiar self-dual instanton solutions,

$$
F_{i j}={ }^{\star} F_{i j} \quad i, j=1, \ldots, 4
$$

In five spacetime dimensions, these are localized solitonic particles. They carry charge $k \in \mathbf{Z}$ under the global $U(1)_{I}$ symmetry with conserved current $J \sim{ }^{\star} \operatorname{Tr} F \wedge F[19]$, and have mass

$$
M_{\mathrm{inst}}=\frac{8 \pi^{2} k}{e^{2}}
$$

The self-duality equations (2.2) have a moduli space $\mathcal{M}$ of solutions, of real dimension $\operatorname{dim}(\mathcal{M})=4 k N$. Away from singularities, the low-energy dynamics is well described by geodesic motion on $\mathcal{M}$ and this provides a good starting point for understanding the quantization of these objects. There is an elegant and convenient way of packaging this dynamics in terms of the ADHM matrix model which we shall review in Section 3.

\footnotetext{
${ }^{2}$ We choose anti-hermitian generators of the Lie algebra satisfying $\left[T_{a}, T_{b}\right]=f_{a b}^{c} T_{c}$, normalized such that $\operatorname{Tr}\left(T_{a} T_{b}\right)=-\frac{1}{2} \delta_{a b}$. The field strength is $F_{\mu \nu}^{a}=\partial_{\mu} A_{\nu}^{a}-\partial_{\nu} A_{\mu}^{a}+f_{b c}^{a} A_{\mu}^{b} A_{\nu}^{c}$ and the covariant derivative is $\mathcal{D}_{\mu} \phi^{a}=\partial_{\mu} \phi^{a}+f_{b c}^{a} A_{\mu}^{b} \phi_{c}$. We define $F_{\mu \nu}=F_{\mu \nu}^{a} T_{a}$, etc.
} 
Let us now add to (2.1) the supersymmetric Chern-Simons Lagrangian [19, 20]

$$
\mathcal{L}_{C S}=\frac{c}{4 \pi^{2}} d_{a b c} \phi^{a}\left(-\frac{1}{4} F_{\mu \nu}^{b} F^{c \mu \nu}-\frac{1}{2} \mathcal{D}_{\mu} \phi^{b} \mathcal{D}^{\mu} \phi^{c}\right)+\frac{c}{24 \pi^{2}} \omega(A)
$$

Here $d_{a b c}$ is the totally symmetric tensor

$$
d_{a b c}=\frac{1}{2} \operatorname{Tr}\left(T^{a}\left\{T^{b}, T^{c}\right\}\right) .
$$

The last term $\omega(A)$ is the Chern-Simons five-form

$$
\omega(A)=\epsilon^{\mu \nu \rho \sigma \lambda} \operatorname{Tr}\left(A_{\mu} \partial_{\nu} A_{\rho} \partial_{\sigma} A_{\lambda}-\frac{3 i}{2} A_{\mu} A_{\nu} A_{\rho} \partial_{\sigma} A_{\lambda}-\frac{3}{5} A_{\mu} A_{\nu} A_{\rho} A_{\sigma} A_{\lambda}\right) .
$$

The first and second terms in (2.4) are dictated by supersymmetry [19, 20]. In the presence of the CS term, invariance of the partition function under large gauge transformations imposes the quantization requirement $c \in \mathbf{Z}$.

The CS term couples the gauge field to the instanton current $J$, telling us that instantons now carry non-Abelian gauge charge ${ }^{3}$ [19]. This ensures that the self-dual configurations (2.2) now source $A_{0}$ and $\phi$. We working in static gauge $\partial_{0}=0$, and set $A_{0}=\phi$. It can be shown that the equations of motion are satisfied if the self-dual equations are supplemented by Gauss' law, which can be written as [22]:

$$
\mathcal{D}^{2}\left(\frac{\phi^{a}}{e^{2}}+\frac{c}{8 \pi^{2}} d_{a b c} \phi^{b} \phi^{c}\right)=\frac{c}{16 \pi^{2}} d_{a b c} F_{i j}^{b} F_{i j}^{c}
$$

To our knowledge, generic solutions to this equation have not been studied in detail, although it is expected that there exists a unique solution for each self-dual configuration. If this is indeed the case, one can show that the mass (2.3) of the instanton remains unchanged in the presence of a CS interaction. We stress that the presence of the $\phi$ field, with interactions (2.4), is crucial if the mass of the instanton is to remain unchanged. Indeed, if one considers the $U(N)$ CS theory without the $\phi$ field then no solution exists: the CS term induces an electric charge, forcing the instanton to large size [7]. We will return to this case at the end of Section 3.

The question that we want to ask is: how do the instantons move in the presence of a CS interaction? Our strategy for answering this question is simple, but indirect. We will replace the Chern-Simons interactions (2.4) with something that we understand well: fermions.

\footnotetext{
${ }^{3}$ When $\langle\phi\rangle \neq 0$, instantons can also carry Abelian gauge charge, even in the absence of a ChernSimons term [21].
} 


\section{Integrating Out Hypermultiplet Fermions}

Instead of working directly with the Chern-Simons Lagrangian (2.4), we instead set $c_{\text {classical }}=0$ and introduce $N_{f}$ hypermultiplets, each of mass $m$, in the fundamental representation of $G$. Each of these hypermultiplets contains two complex scalars and a single Dirac fermion $\Psi$, which satisfies

$$
\not D \Psi-(i \phi+m) \Psi=0
$$

(The unfamiliar factor of $i$ in front of $\phi=\phi^{a} T^{a}$ arises because we are working with anti-Hermitian generators $T^{a}$ ). Integrating out these fermions induces the interaction (2.4), with coefficient $[23,24]$

$$
c=-\frac{N_{f}}{2} \operatorname{sign}(m)
$$

(Here we've assumed $\operatorname{Tr} \phi=0$ in vacuum; otherwise it is $\operatorname{sign}(N m+i \operatorname{Tr} \phi)$ which is the relevant quantity). As well as the induced Chern-Simons term, there is also a finite renormalization of the gauge coupling [20]

$$
\frac{1}{e^{2}} \longrightarrow \frac{1}{e_{\mathrm{ren}}^{2}}=\frac{1}{e^{2}}+\frac{c}{4 \pi^{2}} m
$$

Notice that this shifts the instanton mass $M_{\mathrm{inst}} \rightarrow M_{\mathrm{inst}}-N_{f}|m|$, an effect first pointed out in $[25]$.

All other effects of integrating out the hypermultiplets are suppressed by powers of $1 / m$. By taking the limit $m \rightarrow \infty$, keeping $e_{\text {ren }}^{2}$ fixed, we ensure that the resulting theory is the same as that described by Lagrangian (2.1) and (2.4). We will now study the effects of the fermions on the instanton dynamics.

\section{Instanton Dynamics}

The low-energy dynamics of instantons in a $U(N)$ gauge theory is succinctly captured in the ADHM matrix model ${ }^{4}[15,16]$. For a detailed review, see [27]. In this section, we first review the ADHM formalism for instanton dynamics in the Yang-Mills theory described by the Lagrangian (2.1). We then derive the modification of the dynamics once we add the CS interaction (2.4).

\footnotetext{
${ }^{4}$ The fact that the ADHM matrix model describes $U(N)$ instantons, rather than $S U(N)$ instantons, is clear from the brane picture [16] and also from the generalization to non-commutative backgrounds [26]. While this distinction is irrelevant for pure Yang-Mills theory, it becomes important in the presence of fundamental matter or, as stressed in [7], Chern-Simons interactions.
} 
The dynamics of $k$ instantons in $d=4+1$ dimensional $U(N)$ Yang-Mills theory can be formulated in terms of a $U(k)$ gauge theory on the $d=0+1$ instanton worldline. When the 5 d theory has $\mathcal{N}=1$ supersymmetry (i.e. 8 supercharges), the instanton worldline theory has $\mathcal{N}=(0,4)$ supersymmetry $^{5}$. One introduces an auxiliary $U(k)$ vector multiplet which contains a $U(k)$ Hermitian worldline gauge field $\alpha$ together with a single real adjoint scalar $\sigma$. The dynamical fields, which parameterize the instanton moduli space, include two complex $k \times k$ matrices $W$ and $Z$, each of which transforms in the adjoint of the $U(k)$ gauge group. There are a further $N k$-vectors $\varphi_{i}$, $i=1, \ldots, N$, transforming in the fundamental representation of $U(k)$, and $N k$-vectors $\tilde{\varphi}_{i}$ transforming in the anti-fundamental representation.

The low-energy dynamics of instantons is described the Lagrangian

$$
\begin{aligned}
L=\operatorname{Tr} & \left(\left|\mathcal{D}_{t} Z\right|^{2}+\left|\mathcal{D}_{t} W\right|^{2}+\left|\mathcal{D}_{t} \varphi_{i}\right|^{2}+\left|\mathcal{D}_{t} \tilde{\varphi}_{i}\right|^{2}\right) \\
& -\operatorname{Tr}\left(|[\sigma, Z]|^{2}+|[\sigma, W]|^{2}\right)-\varphi_{i}^{\dagger} \sigma^{2} \varphi_{i}-\tilde{\varphi}_{i} \sigma^{2} \tilde{\varphi}_{i}^{\dagger}
\end{aligned}
$$

where $\mathcal{D}_{t} Z=\dot{Z}-i[\alpha, Z]$, and similar for $W$, while $\mathcal{D}_{t} \varphi_{i}=\dot{\varphi}_{i}-i \alpha \varphi_{i}$ and $\mathcal{D}_{t} \tilde{\varphi}_{i}=$ $\dot{\tilde{\varphi}}_{i}+i \tilde{\varphi}_{i} \alpha$. The fields are subject to constraints which arise from the D and F-terms in the vector multiplet. They are given by

$$
\begin{aligned}
{\left[Z, Z^{\dagger}\right]+\left[W, W^{\dagger}\right]+\varphi_{i} \varphi_{i}^{\dagger}-\tilde{\varphi}_{i}^{\dagger} \tilde{\varphi}_{i}=0 } \\
{[Z, W]+\varphi_{i} \tilde{\varphi}_{i}=0 }
\end{aligned}
$$

After quotienting by the gauge action $U \in U(k)$

$$
Z \rightarrow U Z U^{\dagger} \quad, \quad W \rightarrow U W U^{\dagger} \quad, \quad \varphi_{i} \rightarrow U \varphi_{i} \quad, \quad \tilde{\varphi}_{i} \rightarrow \tilde{\varphi}_{i} U^{\dagger}
$$

the scalars provide coordinates on the instanton moduli space $\mathcal{M}$. Given a suitable parametrization of the constraints (3.12), the first line in (3.11) reduces to the hyperKähler metric on $\mathcal{M}$.

The auxiliary field $\sigma$ that appears on the second line of (3.11) plays no role in the purely bosonic theory (at least if $\langle\phi\rangle=0$ ). However the $\mathcal{N}=(0,4)$ ADHM Lagrangian also comes with a number of Grassmann valued fields. These describe the zero modes of the adjoint Dirac fermion $\lambda$ and live in the tangent bundle of $\mathcal{M}$. They won't play an important role in our story, so we do not describe them here. When they are included, integrating out $\sigma$ induces four-fermi terms in the instanton action.

\footnotetext{
${ }^{5}$ This arises from the dimensional reduction of the chiral $\mathcal{N}=(0,4)$ superalgebra in two dimensions. It is sometimes called $N=4 B$ supersymmetry to distinguish it from the $\mathcal{N}=(2,2)$ superalgebra.
} 


\section{Integrating out Hypermultiplet Fermions}

Let us now examine the effect of the supplementary fermions $\Psi$ that we introduced solely for the purpose of inducing Chern-Simons interactions in 5d. We start by studying solutions to the Dirac equation (2.8) in the background of a self-dual instanton $F={ }^{\star} F$ configuration. Of the full spectrum of solutions, only the zero modes will prove important. We discuss these first, and then explain why the non-zero modes are unimportant. We choose the basis of $4 \times 4$ gamma matrices,

$$
\gamma^{0}=\left(\begin{array}{cc}
-i & 0 \\
0 & +i
\end{array}\right) \quad, \quad \gamma^{i}=\left(\begin{array}{cc}
0 & \sigma^{i} \\
\sigma^{i} & 0
\end{array}\right) \quad, \quad \gamma^{4}=\left(\begin{array}{cc}
0 & i \\
-i & 0
\end{array}\right)
$$

with $\sigma^{i}, i=1,2,3$, the Pauli matrices. For each $\Psi$, the "zero mode" solutions arise from the ansatz $\Psi^{T}=\left(e^{+i m t} \psi_{+}, e^{-i m t} \psi_{-}\right)$where $\psi_{ \pm}$are two-component spinors that satisfy

$$
\begin{aligned}
& \not D \psi_{-} \equiv \sigma_{i} \mathcal{D}_{i} \psi_{-}+i \mathcal{D}_{4} \psi_{-}=0 \\
& \bar{D} \psi_{+} \equiv \sigma_{i} \mathcal{D}_{i} \psi_{+}-i \mathcal{D}_{4} \psi_{+}=0
\end{aligned}
$$

In the self-dual instanton background, standard index theorems state that $\not D$ has $2 k$ complex zero modes, while $\overline{D D}$ has none. We introduce the Grassmann collective coordinates for these modes: $\xi_{-}^{m}(t)$ and $\tilde{\xi}_{-}^{m}(t)$, with $m=1, \ldots, k$. In the language of the ADHM construction, these Grassmann collective coordinates live in a single $\mathcal{N}=(0,4)$ fermi multiplet. $\xi_{-}$transforms in the $\mathbf{k}$ representation of $U(k)$, while $\tilde{\xi}_{-}$transforms in the $\overline{\mathbf{k}}$ representation. The worldline Lagrangian governing their dynamics is,

$$
L_{\mathrm{fermi}}=\bar{\xi}_{-}\left(i D_{t}+\sigma+m\right) \xi_{-}+\tilde{\xi}_{-}\left(i D_{t}+\sigma+m\right) \overline{\tilde{\xi}}_{-}
$$

There are no further constraints on $\xi_{-}$and $\tilde{\xi}_{-}$. (See, for example Section VI of [27], where $\xi_{-}$and $\tilde{\xi}_{-}$are referred to as $\mathcal{K}$ and $\left.\tilde{\mathcal{K}}\right)$.

We now come to quantize these Grassmann collective coordinates. Firstly note that, although we have been referring to them as "zero modes", this is a slight misnomer. As is clear from (3.16), they are excited at a cost of energy equal to $|m|$. This is unsurprising, since they arose from the $5 \mathrm{~d}$ fermion $\Psi$ with mass $m$. Quantization gives rise to the usual ordering ambiguity. Comparison with renormalization of $e^{2}$ suggests that the correct quantization takes the ground state to have energy $-|m|[25]$.

Our interest here lies in the effect of these zero modes on the low-energy bosonic dynamics of the instantons. To see this effect, we integrate out $\xi_{-}^{m}$ and $\tilde{\xi}_{-}^{m}$ at one loop. 
A standard computation of the determinants, given in [17], shows that each of these fermions contributes a term,

$$
L_{\text {eff }}=\log \left[\frac{\operatorname{det}\left(i \partial_{t}+\alpha+\sigma+m\right)}{\operatorname{det}\left(i \partial_{t}+m\right)}\right]=\frac{1}{2} \operatorname{sign}(m) \operatorname{Tr}(\alpha+\sigma)
$$

The $\operatorname{Tr}(\alpha)$ term is a quantum mechanical Chern-Simons coupling. Invariance under large gauge transformations requires that the coefficient is integer valued, which is indeed the case because we have integrated out two fermions, $\xi_{-}^{m}$ and $\tilde{\xi}_{-}^{m}$. The $\operatorname{Tr}(\sigma)$ term is a linear potential for the auxiliary field; we will see the meaning of this shortly. Integrating out all $N_{f}$ hypermultiplet zero modes, and comparing to (2.9), we arrive at the main result of the paper: the low-energy dynamics of instantons in the theory with 5d CS interactions (2.4) is governed by the ADHM CS matrix model ${ }^{6}$,

$$
\begin{gathered}
L=\operatorname{Tr}\left(\left|\mathcal{D}_{t} Z\right|^{2}+\left|\mathcal{D}_{t} W\right|^{2}+\left|\mathcal{D}_{t} \varphi_{i}\right|^{2}+\left|\mathcal{D}_{t} \tilde{\varphi}_{i}\right|^{2}-2 c(\alpha+\sigma)\right) \\
-\operatorname{Tr}\left(|[\sigma, Z]|^{2}+|[\sigma, W]|^{2}\right)-\varphi_{i}^{\dagger} \sigma^{2} \varphi_{i}-\tilde{\varphi}_{i} \sigma^{2} \tilde{\varphi}_{i}^{\dagger}
\end{gathered}
$$

\section{Why Only Zero Modes Matter}

In deriving the Lagrangian (3.18), we have integrated out only the zero modes on the instanton worldline, while ignoring the infinite tower of higher solutions to the Dirac equation. We now show that this is consistent. The key point is that higher excitations of fermions come in pairs, with energy $\pm E$ :

$$
\left(\begin{array}{cc}
0 & \not D \\
\overline{D D} & 0
\end{array}\right)\left(\begin{array}{l}
\psi_{+} \\
\psi_{-}
\end{array}\right)=E\left(\begin{array}{c}
\psi_{+} \\
\psi_{-}
\end{array}\right) \Rightarrow\left(\begin{array}{cc}
0 & \not D \\
\bar{D} & 0
\end{array}\right)\left(\begin{array}{c}
\psi_{+} \\
-\psi_{-}
\end{array}\right)=-E\left(\begin{array}{c}
\psi_{+} \\
-\psi_{-}
\end{array}\right)
$$

Contributions to the Chern-Simons term on the instanton worldline cancel between each pair. To see this, we write the general eigenfunction as $\Psi^{T}=\left(\psi_{-} \zeta_{-}, \psi_{+} \zeta_{+}\right)$ and promote $\zeta_{ \pm}$to time-dependent Grassmann fields. The action for these objects is schematically

$$
L_{\text {non-zero-modes }}=\bar{\zeta}_{+}\left(i D_{t}-m\right) \zeta_{+}+\bar{\zeta}_{-}\left(i D_{t}+m\right) \zeta_{-}+E\left(\bar{\zeta}_{+} \zeta_{-}+\bar{\zeta}_{-} \zeta_{+}\right)
$$

which is schematic only in the sense that we have dropped overall coefficients that arise from the overlap of the eigenfunctions. Integrating out the non-zero modes now gives us a determinant of the form

$$
\operatorname{det}\left(\begin{array}{cc}
i D_{t}-m & E \\
E & i D_{t}+m
\end{array}\right)=\operatorname{det}\left(i D_{t}+\sqrt{m^{2}+E^{2}}\right) \operatorname{det}\left(i D_{t}-\sqrt{m^{2}+E^{2}}\right)
$$

${ }^{6}$ This Lagrangian appears to differ by the a factor of 2 in the coefficient of the Chern-Simons term with the result of $[18]$. 
We see that the effective mass of these objects is $\pm \sqrt{m^{2}+E^{2}}$, leading to a cancellation due to the presence of the $\operatorname{sign}(m)$ term in (3.17). In the limit $m \rightarrow \infty$, these non-zero modes leave no trace of their existence on the vortex dynamics.

\section{An Example: A Single Instanton}

Let us study the dynamics (3.18) for a single $k=1$ instanton. In this case $Z$ and $W$ describe the trivial centre of mass motion and may be ignored. The remaining $4(N-1)$ collective coordinates describe the scale size $\rho$ and the $U(N)$ orientation of the instanton. In the ADHM formalism, these are captured by $2 N$ complex scalars $\varphi_{i}$ and $\tilde{\varphi}_{i}, i=1 \ldots, N$, subject to the constraints arising from (3.12)

$$
\sum_{i}\left|\varphi_{i}\right|^{2}-\left|\tilde{\varphi}_{i}\right|^{2}=\sum_{i} \varphi_{i} \varphi_{i}=0
$$

and modulo the $U(1)$ gauge action: $\varphi_{i} \rightarrow e^{i \theta} \varphi_{i}$ and $\tilde{\varphi}_{i} \rightarrow e^{-i \theta} \tilde{\varphi}_{i}$. These define the moduli space $\mathcal{M} \cong T^{\star} \mathbf{C} \mathbf{P}^{N-1}$, the cotangent bundle of $\mathbf{C P}^{N-1}$, with vanishing Kähler class. The singularity at the origin of $\mathcal{M}$ corresponds to the singular, small instanton. Away from the singularity, the scale size $\rho$ of the instanton is identified as

$$
\sum_{i}\left|\varphi_{i}\right|^{2}+\left|\tilde{\varphi}_{i}\right|^{2}=\frac{16 \pi^{2}}{e^{2}} \rho^{2}
$$

To illustrate the various terms in the Lagrangian (3.18), let us first ignore the auxiliary field $\sigma$ and concentrate only on the kinetic terms. They are given by,

$$
L=\left|\mathcal{D}_{t} \varphi_{i}\right|^{2}+\left|\mathcal{D}_{t} \tilde{\varphi}_{i}\right|^{2}-2 c \alpha=\left|\dot{\varphi}_{i}\right|^{2}+\left|\dot{\tilde{\varphi}}_{i}\right|^{2}-\alpha(j+2 c)+\frac{16 \pi^{2}}{e^{2}} \alpha^{2} \rho^{2}
$$

where $j=i \sum_{i}\left[\left(\dot{\varphi}_{i}^{\dagger} \varphi_{i}-\varphi_{i}^{\dagger} \dot{\varphi}_{i}\right)-\left(\dot{\tilde{\varphi}}_{i}^{\dagger} \tilde{\varphi}_{i}-\tilde{\varphi}_{i}^{\dagger} \dot{\tilde{\varphi}}_{i}\right)\right]$ is the $U(1)$ gauge current. To perform the $U(1)$ quotient, we simply integrate out the gauge field $\alpha$. The resulting Lagrangian is given by,

$$
L=\left[\left|\dot{\varphi}_{i}\right|^{2}+\left|\dot{\tilde{\varphi}}_{i}\right|^{2}-\frac{e^{2} j^{2}}{64 \pi^{2} \rho^{2}}\right]-\frac{e^{2}}{16 \pi^{2}} \frac{c j}{\rho^{2}}-\frac{e^{2} c^{2}}{16 \pi^{2} \rho^{2}}
$$

The first three terms in square brackets are quadratic in velocities: they implicitly define the metric on the instanton moduli space. (An explicit parametrization of the constraints, and hence the metric, was given in the $N=2$ case in [28]). The fourth term, linear in velocity, is the induced magnetic field on the moduli space. Yet there is also a potential term, of order $c^{2}$, which forces the instanton to large size $\rho \rightarrow \infty$. This term is cancelled by the linear potential for $\sigma$. Indeed, solving (3.18) for $\sigma$ gives,

$$
\sigma=\frac{-c}{\left|\varphi_{i}\right|^{2}+\left|\tilde{\varphi}_{i}\right|^{2}}
$$


which, after substitution, cancels the last term in (3.24). It is simple to check that a similar phenomenon happens for the general non-Abelian matrix model (3.18), with the auxiliary field $\sigma$ cancelling an effective potential on $\mathcal{M}$ induced by the CS term $c \alpha$.

We note that the interplay between the potential generated by the CS term and the linear $\sigma$ potential in the instanton theory mimics a similar interplay in $5 \mathrm{~d}$. Without the scalar field $\phi$, the $U(N)$ CS term generates a potential for the instanton, pushing it to large size. This potential is cancelled by the scalar field $\phi$ which adjusts so that the instanton once again saturates the BPS bound (2.3) for all scale sizes $\rho$. Given this correspondence, one might wonder whether the $U(N)$ theory without $\phi$ is captured by the ADHM model without $\sigma$. However, this does not appear to be the case. One can compute the potential on the instanton moduli space generated by the CS coupling (see, for example [7]). In the $U(2)$ theory, the functional form of this potential agrees with that in (3.24), but the numerical coefficient differs.

\section{A Variation on the Theme: $S U(N)$ Instantons}

The ADHM matrix model describes instantons in $U(N)$ gauge theory with the scalar field $\phi$. For instantons in pure $S U(N)$ Yang-Mills theory (without the scalar field $\phi$ ) we now derive a more geometrical, albeit somewhat implicit, description of the dynamics. Our strategy is as before: we introduce a fermion of mass $m$ which, when integrated out, induces a 5d CS term. These fermions also give rise to a number of instanton zero modes. These define a bundle over the instanton moduli space $\mathcal{M}$, commonly referred to as the index bundle [29]. As we adiabatically change the instanton configuration, and hence move in $\mathcal{M}$, the zero modes undergo a holonomy described by a connection $\omega$ over $\mathcal{M}$. We denote the Grassmann valued coordinates of the fibre as $\xi^{m}$. The appropriate covariant derivative for these collective coordinates is given by,

$$
\mathcal{D}_{t}=\partial_{t} \xi^{m}+\left(\omega_{a}\right)_{n}^{m} \dot{X}^{a} \xi^{n}
$$

where $X^{a}$ are coordinates on $\mathcal{M}$. Comparing with the ADHM description (3.16), we see that the index bundle in that case arose because the Grassmann collective coordinates were charged under the auxiliary $U(k)$ gauge field. In the present case, the description of the index bundle is more geometric. As shown in [14], integrating out the $\xi^{m}$ induces a sigma-model CS term,

$$
L_{C S}=\frac{1}{2} \operatorname{sign}(m) \operatorname{Tr}\left(\omega_{a}\right) \dot{X}^{a}
$$

This is equivalent to a magnetic field $B=\operatorname{Tr} d \omega$ over the instanton moduli space. This is simply the first Chern character of the index bundle. We conclude that the lowenergy dynamics of instantons is described by geodesic motion on $\mathcal{M}$, modified by the presence of this magnetic field. 
Let's look at some examples of this procedure. Consider firstly the effect of adding a massive fermion $\lambda$ in the adjoint representation of $S U(N)$. This does not contribute to a $5 \mathrm{~d}$ CS term because $d_{a b c}=0$ for real representations. We can also see this from the perspective of the instanton moduli space. For an adjoint fermion, index theorems tell us that there exist $2 k N$ complex fermi zero modes. These can be mapped to the bosonic zero modes of the instanton, which ensures that they live in the tangent bundle of $\mathcal{M}$. As explained in [14], integrating out these modes gives rise to a magnetic field on $\mathcal{M}$ which is equal to the first Chern character. Yet the instanton moduli space is hyperKähler, and hence Calabi-Yau, and its first Chern character vanishes. We thus see from both $5 \mathrm{~d}$ and $1 \mathrm{~d}$ points of view that integrating out adjoint fermions does not affect the instanton dynamics.

Let us now consider a massive fermion $\Psi$ in the fundamental representation of $S U(N)$.

For $N \geq 3$, integrating out this fermion in $5 \mathrm{~d}$ induces a CS term with $c=-\frac{1}{2} \operatorname{sign}(m)$. The instanton picks up $2 k$ fermi zero modes which live in an index bundle with a $U(k)$ connection. Integrating out these zero modes in the worldline theory induces a magnetic field over the moduli space $\mathcal{M}$, equal to the first Chern character of the index bundle. The low-energy dynamics of the instantons is described by geodesic motion on $\mathcal{M}$ in the presence of this magnetic field.

For $S U(2)$, no CS term is generated in $5 \mathrm{~d}$ because $d_{a b c}=0$ for all representations. Once again, we can see this from the perspective of instanton zero modes. The index bundle for $S U(2)$ instantons has a $O(k)$ connection, rather than a $U(k)$ connection [29], ensuring that the Chern class vanishes and no magnetic field is generated on $\mathcal{M}$.

\section{Acknowledgement}

We would like to thank Ben Allanach, Ki-Myeong Lee and Piljin Yi for useful discussions. BC is supported by an STFC studentship. DT is supported by the Royal Society. 


\section{References}

[1] M. F. Atiyah and N. S. Manton, "Skyrmions From Instantons," Phys. Lett. B 222, 438 (1989).

[2] T. Sakai and S. Sugimoto, "Low energy hadron physics in holographic QCD," Prog. Theor. Phys. 113, 843 (2005) [arXiv:hep-th/0412141].

[3] T. Sakai and S. Sugimoto, "More on a holographic dual of QCD," Prog. Theor. Phys. 114, 1083 (2006) arXiv:hep-th/0507073.

[4] C. T. Hill, "Topological solitons from deconstructed extra dimensions," Phys. Rev. Lett. 88, 041601 (2002) [arXiv:hep-th/0109068]; C. T. Hill and C. K. Zachos, "Dimensional deconstruction and Wess-Zumino-Witten terms," Phys. Rev. D 71, 046002 (2005) [arXiv:hep-th/0411157].

[5] D. T. Son and M. A. Stephanov, "QCD and dimensional deconstruction," Phys. Rev. D 69, 065020 (2004) [arXiv:hep-ph/0304182].

[6] M. Eto, M. Nitta, K. Ohashi and D. Tong, "Skyrmions from instantons inside domain walls," Phys. Rev. Lett. 95, 252003 (2005) [arXiv:hep-th/0508130].

[7] H. Hata, T. Sakai, S. Sugimoto and S. Yamato, "Baryons from instantons in holographic QCD," arXiv:hep-th/0701280.

[8] D. K. Hong, M. Rho, H. U. Yee and P. Yi, "Chiral dynamics of baryons from string theory," Phys. Rev. D 76, 061901 (2007) [arXiv:hep-th/0701276].

[9] D. K. Hong, M. Rho, H. U. Yee and P. Yi, "Dynamics of Baryons from String Theory and Vector Dominance," JHEP 0709, 063 (2007) [arXiv:0705.2632 [hep-th]].

[10] H. Hata and M. Murata, "Baryons and the Chern-Simons term in holographic QCD with three flavors," arXiv:0710.2579 [hep-th].

[11] H. Hata, M. Murata and S. Yamato, "Chiral currents and static properties of nucleons in holographic QCD," arXiv:0803.0180 [hep-th].

[12] J. Goldstone and F. Wilczek, "Fractional Quantum Numbers On Solitons," Phys. Rev. Lett. 47, 986 (1981).

[13] Y. H. Chen and F. Wilczek, "Induced Quantum Numbers in Some (2+1)-Dimensional Models," in "Fractional statistics and anyon superconductivity," World Scientific (1990).

[14] B. Collie and D. Tong, "The Dynamics of Chern-Simons Vortices", to appear.

[15] M. F. Atiyah, N. J. Hitchin, V. G. Drinfeld and Yu. I. Manin, "Construction of instantons," Phys. Lett. A 65, 185 (1978).

[16] M. R. Douglas, "Branes within branes," arXiv:hep-th/9512077. 
[17] T. Banks, N. Seiberg and E. Silverstein, "Zero and one-dimensional probes with $N=8$ supersymmetry," Phys. Lett. B 401, 30 (1997) [arXiv:hep-th/9703052].

[18] S. Kim, K. M. Lee and S. Lee, "Dyonic Instantons in 5-dim Yang-Mills Chern-Simons Theories," arXiv:0804.1207 [hep-th].

[19] N. Seiberg, "Five dimensional SUSY field theories, non-trivial fixed points and string dynamics," Phys. Lett. B 388, 753 (1996) [arXiv:hep-th/9608111].

[20] K. A. Intriligator, D. R. Morrison and N. Seiberg, "Five-dimensional supersymmetric gauge theories and degenerations of Calabi-Yau spaces," Nucl. Phys. B 497, 56 (1997) [arXiv:hep-th/9702198].

[21] N. D. Lambert and D. Tong, "Dyonic instantons in five-dimensional gauge theories," Phys. Lett. B 462, 89 (1999) [arXiv:hep-th/9907014].

[22] S. Kim and S. Lee, "The Geometry of Dyonic Instantons in 5-dimensional Supergravity," arXiv:0712.0090 [hep-th].

[23] A. J. Niemi and G. W. Semenoff, "Axial Anomaly Induced Fermion Fractionization And Effective Gauge Theory Actions In Odd Dimensional Space-Times," Phys. Rev. Lett. 51, 2077 (1983).

[24] E. Witten, "Phase Transitions In M-Theory And F-Theory," Nucl. Phys. B 471, 195 (1996) [arXiv:hep-th/9603150].

[25] O. Aharony, A. Hanany and B. Kol, "Webs of $(p, q)$ 5-branes, five dimensional field theories and grid diagrams," JHEP 9801, 002 (1998) [arXiv:hep-th/9710116].

[26] N. Nekrasov and A. S. Schwarz, "Instantons on noncommutative $R^{* *} 4$ and $(2,0)$ superconformal six dimensional theory," Commun. Math. Phys. 198, 689 (1998) [arXiv:hep-th/9802068].

[27] N. Dorey, T. J. Hollowood, V. V. Khoze and M. P. Mattis, "The calculus of many instantons," Phys. Rept. 371, 231 (2002) [arXiv:hep-th/0206063].

[28] K. M. Lee, D. Tong and S. Yi, "The moduli space of two U(1) instantons on noncommutative $R^{4}$ and $R^{3} \times S^{1}$," Phys. Rev. D 63, 065017 (2001) [arXiv:hep-th/0008092].

[29] N. S. Manton and B. J. Schroers, "Bundles over moduli spaces and the quantization of BPS monopoles," Annals Phys. 225, 290 (1993). 